\title{
La cultura sobre seguridad informática en las redes sociales: el caso de los estudiantes de la Preparatoria de San Diego Cuentla, México
}

The culture on information security in social networks: the case of students of San Diego Cuentla High School, Mexico A cultura de segurança nas redes sociais: o caso dos alunos da Escola de San Diego Cuentla, México DOI: http://dx.doi.org/10.23913/ricsh.v6i11.106

Irma Arellano Martínez Universidad Autónoma del Estado de México, Centro Universitario UAEM Temascaltepec, México irarma_2@hotmail.com

\section{Resumen}

El objetivo de la investigación fue conocer la cultura en seguridad informática con respecto al riesgo que genera el uso de las redes sociales en estudiantes de la Preparatoria 193 ubicada en San Diego Cuentla, Estado de México, específicamente en 44 alumnos de ambos sexos de primer grado, turno matutino, con 14 y 15 años de edad.

Para ello se utilizó metodología descriptiva y un cuestionario de diez preguntas cerradas. posteriormente se procesó la información en el paquete SPSS calculando las frecuencias de uso, lo que permitió conocer cuáles eran las redes más populares entre los estudiantes y sus riesgos.

La gran mayoría de los alumnos (91\%) pertenece a alguna red social, especialmente Facebook con $48 \%$; asimismo, $59 \%$ conoce los controladores de configuración, $54 \%$ comparte información sólo con sus amigos, $36 \%$ nunca cambia sus contraseñas y $95 \%$ no publica dónde se ubica y qué hace. Por otro lado, $82 \%$ dijo que no se cerciora de haber cerrado su sesión, $73 \%$ que sí sabe de los riesgos a los que está expuesto y 66 \% que ya conoce los delitos que se cometen en las redes sociales, en especial ciberbullyng (32\%).

Se detectó que a los estudiantes les falta cultura sobre el uso de las redes sociales, lo cual los pone en situación de riesgo. 
Palabras clave: cultura, seguridad informática, factor de riesgo, redes sociales, estudiantes, preparatoria.

\section{Abstract}

The objective of the research was to know the culture in information security with respect to the risk that the use of social networks in students of the High School \#193 located in San Diego Cuentla, State of Mexico, specifically in 44 students of both sexes of Freshmen, morning shift, with 14 and 15 years old.

For this purpose we used descriptive methodology and a questionnaire of ten closed questions. Subsequently the information was processed in the SPSS package calculating frequencies of use, allowing you to know what were the most popular networks between students and their risks.

The vast majority of students (91\%) belongs to a social network, especially Facebook with $48 \%$; likewise, 59\% known configuration drivers, 54\% share information only with their friends, 36\% never changes their passwords and 95\% do not publish where they are located and what they do. On the other hand, $82 \%$ said that not sure having closed its session, $73 \%$, who knows of the risks to which it is exposed and 66\% that already knows the crimes that are committed in social networks, especially ciberbullyng (32\%).

We detected that the students have a lack culture on the use of social networks, which puts them at risk.

Key words: culture, computer security, risk factor, social network, students, high school.

\section{Resumo}

O objetivo da pesquisa foi conhecer a cultura em segurança de computadores em relação ao risco gerado pelo uso de redes sociais alunos da Escola 193 localizadas em San Diego Cuentla, Estado do México, especificamente em 44 estudantes de ambos os sexos de primeiro grau , turno da manhã, com 14 e 15 anos de idade.

Por esta metodologia descritiva e questionário de dez perguntas fechadas foi usado. posteriormente as informações no pacote SPSS foi processado através do cálculo da 
frequência de utilização, permitindo saber o que as redes mais populares entre os estudantes e seus riscos eram.

A grande maioria dos alunos (91\%) pertence a uma rede social, especialmente o Facebook, com 48\%; Além disso, 59\% conhecem a configuração motoristas, 54\% compartilha informações apenas com amigos, 36\% nunca mudam suas senhas e $95 \%$ não publica onde ele está localizado eo que ele faz. Por outro lado, $82 \%$ disseram que não tem certeza de ter desconectado, $73 \%$ que sabem dos riscos aos quais está exposta e $66 \%$ já conhece os crimes cometidos em redes sociais, especialmente ciberbullyng ( $32 \%)$.

Verificou-se que os alunos não têm cultura sobre o uso de redes sociais, o que os coloca em risco.

Palavras-chave: cultura, segurança, fatores de risco, as redes sociais, estudantes, escola.

Fecha recepción: Junio 2016 Fecha aceptación: Diciembre 2016

\section{Introducción}

El internet ha crecido cada vez más en todo el mundo y, por tanto, también el riesgo de que sea mal utilizado debido a la falta de información.

La red social más grande en nuestro país sigue siendo Facebook, seguida de WhatsApp, donde están 8 de cada 10 internautas, quienes en promedio están inscritos en cinco redes sociales (AMIPCI, 2016).

El manejo de las redes sociales ofrece múltiples ventajas, entre las cuales está que facilita la búsqueda de información y la interacción entre los estudiantes; por otro lado, una desventaja sería que pueden ser víctimas de acoso. El uso de la red social Facebook, la más popular entre los estudiantes, incrementa los riesgos a los que se exponen.

La Escuela Preparatoria de San Diego, caso en estudio, se ubica sobre la carretera TolucaZihuatanejo $\mathrm{Km} 75.5$ con un promedio de 240 alumnos, a quienes se les aplicó un instrumento que demostró que $91 \%$ usa al menos una red social. 


\section{VENTAJAS Y DESVENTAJAS DE LAS REDES SOCIALES}

\section{DESVENTAJAS}

El libro Recursos tecnológicos en contextos educativos (Maria, Cristina, y Jesús, 2016) enumera las siguientes desventajas:

- Imparcialidad, ya que se puede crear fácilmente una identidad falsa.

- Falta de veracidad, pues la información disponible no siempre es fidedigna.

- Falta de productividad, por los altos niveles de dependencia y aislamiento.

- Numerosos casos de pornografía infantil y pedofilia.

- Acoso por parte de personas conocidas o desconocidas.

- Incumplimiento de leyes, incluso de manera inintencionada.

\section{VENTAJAS}

La Universidad Católica de Valencia (2016) menciona las siguientes ventajas:

- Facilita la interacción entre los estudiantes: las redes sociales pueden incrementar la colaboración entre los diferentes compañeros de clase, ya que es muy fácil establecer vías de comunicación y diálogo que permitan trabajar en un proyecto colaborativo.

- Facilita la búsqueda de información: la mayoría de blogs y páginas web que publican contenido de valor lo difunden en las redes sociales, por ese motivo se puede encontrar información de altísimo valor que ayuda en la preparación de trabajos académicos.

- Facilita el compartir recursos y contenidos: los medios sociales son un fantástico vehículo para que cualquier alumno pueda difundir recursos o contenidos, y así colaborar a la creación de una biblioteca.

- Genera debates y actividades sobre una temática determinada: antes de que aparecieran las redes sociales se debatía en internet mediante foros, pero desde su llegada es la mejor herramienta de discusión. Existen redes sociales como Twitter, donde se pueden seguir discusiones según el hashtag, de esta forma cualquier persona puede opinar sobre el tema.

- Permite la comunicación con profesionales de cualquier materia: a través de las redes los alumnos pueden comunicarse con profesores de su propia institución educativa $u$ otras distintas en cuestión de segundos. 
- Agiliza el proceso de aprendizaje: los profesores utilizan cada vez más las redes como herramienta, lo que favorece el aprendizaje.

- Incentiva y fomenta la investigación: el acceso a grandes volúmenes de información generados por profesores y profesionales de todo el mundo, fomenta la investigación y la profundización de un tema concreto.

\section{REDES SOCIALES MÁS CONOCIDAS}

De acuerdo con Forbes (2016), las redes sociales más conocidas son:

- Facebook

- YouTube

- FacebookMessenger

- Twitter

- Google+

- WhatsApp

- Instagram

- LinkendIn

- Pinterest, etcétera.

\section{RIESGOS EN LAS REDES SOCIALES}

De acuerdo a Ellen, Temmy, y Martin (2014), los riesgos se clasifican en tres categorías:

- La primera describe los riesgos de contenido. Un ejemplo típico de contenido provocador que pueden encontrar los adolescentes en las redes sociales son los mensajes de odio. Estos mensajes pueden ser bastante directos, como actualizaciones de estado o publicaciones de carácter agresivo en el muro de alguien, pero también pueden ser indirectos, por ejemplo, uniéndose a grupos de odio. Los adolescentes necesitan igualmente desarrollar habilidades críticas para juzgar la fiabilidad de la información. La información errónea que podría aparecer en las redes puede ser intencionada, por ejemplo, un cotilleo publicado por otros usuarios, o involuntaria. Esta última puede darse cuando alguien publica una broma que puede ser malinterpretada como información veraz. Entre los ejemplos típicos encontramos artículos de revistas satíricas publicadas en el muro de una red social. 
- La segunda categoría de riesgos incluye los riesgos de contacto, que son aquellos que tienen su origen en el hecho de que las redes sociales puedan utilizarse como herramienta para comunicarse y establecer contacto con otros. Junto a la mensajería instantánea, las redes son los medios más populares utilizados para el ciber-acoso, ya sea a través de chat, mediante la publicación de mensajes ofensivos o creando páginas de grupos de odio. Además, también se pueden utilizar para solicitar servicios sexuales, como se observa en el proceso de captación de menores, donde un adulto con intenciones sexuales logra establecer una relación con un menor a través de Internet. Por otra parte, los usuarios se enfrentan a los riesgos de privacidad dada la gran cantidad de información personal que publican en línea. Asimismo, 29 \% de los adolescentes mantiene un perfil público o ignora la configuración de su privacidad, y $28 \%$ opta por una configuración parcialmente privada para que los amigos de sus amigos pueden ver su perfil.

- La tercera categoría de riesgos contiene los riesgos comerciales. Estos incluyen el uso indebido de datos personales. La información se puede compartir con terceras empresas mediante aplicaciones, del mismo modo que se puede realizar un seguimiento al comportamiento del usuario para ofrecerle anuncios publicitarios y publicidad social orientada a su perfil. Todos estos riesgos constituyen una amenaza, ya que hay estudios que indican que la exposición a los riesgos en línea provoca daños y experiencias negativas en un número importante de casos El acoso en Internet es visto como un importante problema de salud pública, con los agresores haciendo frente a múltiples problemas, entre ellos una mediocre relación entre padres e hijos, consumo de drogas y delincuencia. Además, algunas teorías predicen que los adolescentes son menos propensos a reconocer los riesgos y las futuras consecuencias de sus decisiones. Igualmente, se constató que tienen más dificultad para controlar sus impulsos y poseen niveles más altos de búsqueda de emociones y desinhibición que los adultos. Esto podría aumentar el riesgo que asumen los adolescentes, sobre todo porque publicar fotos e intereses ayuda a crear y revelar la identidad del individuo. 


\section{MATERIALES Y MÉTODOS}

La investigación del presente trabajo se llevó a cabo en la localidad de San Diego Cuentla, en el Municipio San Simón de Guerrero, Estado de México, ubicado a 90 Km de la capital Toluca. La Preparatoria Oficial núm.193 se encuentra ubicada en la carretera TolucaZihuatanejo Km 75 (googlemaps, 2016).

El tamaño de la población de dicha preparatoria asciende a 240 alumnos, pero sólo se tomó en cuenta a los de primer grado: 44 alumnos de ambos sexos en edades de 14 y 15 años (información proporcionada por el director).

El instrumento fueron diez preguntas cerradas previa prueba piloto. Una vez que se corroboró que no había ninguna duda al respecto se procedió a su aplicación. Posteriormente se procesó la información con el paquete de SPSS versión 17, y se calcularon las frecuencias de uso.

\section{RESULTADOS}

- Ítem 1. ¿Tienes una red social?

40 contestaron sí y 4 no, lo que representa 91 y $9 \%$ respectivamente.

- Ítem 2. ¿En qué redes sociales participas?

21 dijeron Facebook, 4 WhatsApp y 15 más de una red social, lo que representa 48, 34 y $9 \%$ respectivamente.

- Ítem 3. ¿Conoces los controles de configuración de tu red social?

26 contestaron no y 18 sí, es decir, 59 y $41 \%$ respectivamente.

- Ítem 4. ¿Cómo compartes tu información?

24 estudiantes contestaron que lo hacen sólo con sus amigos, 10 con el público en general, 6 nunca de manera pública, 3 sólo yo, y 1 con su familia, es decir, 54, 23, 14, 7 y $2 \%$ respectivamente.

- Ítem 5. ¿Cada cuándo cambias tus contraseñas de la red social que usas? 
10 dijeron cada año, 16 no la cambian nunca, 9 cada seis meses y 9 cada mes. Esto significa $36,23,21$ y $20 \%$ respectivamente.

- Ítem 6. ¿Sueles publicar dónde estás y qué haces?

42 dijeron sí y 2 no, lo que significa 95 y $2 \%$ respectivamente.

- Ítem 7. ¿Te cercioras de que hayas cerrado correctamente tu sesión?

36 alumnos contestaron que no y 8 que sí, o sea 82 y $18 \%$ respectivamente.

- Ítem 8. ¿Conoces los riesgos a los que te expones al utilizar las redes sociales?

32 contestaron sí y 12 no, es decir, 73 y $27 \%$ respectivamente.

- Ítem 9. ¿Has escuchado hablar de los delitos que se cometen en las redes sociales?

29 alumnos contestaron sí y 15 no, lo que representa 66 y $34 \%$ respectivamente.

- Ítem 10. ¿Conoces o has escuchado hablar de algún riesgo?

19 dijeron conocer más de un riesgo, 15 mencionaron ciberbullyng, 5 ciber-acoso, 4 que no conocen ninguno y 1 grooming, lo que representa $43,34,12,9$ y $2 \%$ respectivamente.

\section{DISCUSIÓN}

En la presente investigación se encontró que Facebook es la red más utilizada por los estudiantes de la Preparatoria de San Diego Cuentla. Asimismo, las redes más visitadas por los jóvenes y que han crecido más durante los últimos años son: Facebook, MySpace y Twitter (Roxana, Atilio, Vanina, y Florencia, 2010). El rango de edad en el que se suele usar más las redes sociales es de los 11 a los 30 años, lo cual coincide con los resultados de la presente investigación realizada en la preparatoria, cuyos alumnos tienen entre 14 y 15 años de edad.

Según la AMIPCI (2016), las redes sociales tienen a $75 \%$ de los internautas, de los cuales 92 $\%$ están en Facebook. Esto coincide con el resultado de la red social más utilizada por los estudiantes de la preparatoria. 
Asimismo, de acuerdo a los estudios realizados por el Observatorio Nacional de Telecomunicaciones y S. I (ONTSI, 2011), tan sólo 10 \% de los usuarios de las redes sociales las emplea frecuentemente para compartir textos en España. Si se compara esto con la investigación realizada en la preparatoria, el porcentaje es mayor (54\%) pero compartiendo cualquier tipo de información.

Los usuarios no son plenamente conscientes de que sus datos personales serán visibles para cualquier persona (María, Virginia, y Emilio). Si se toma esto en cuenta, los estudiantes de la preparatoria de San Diego Cuentla deben recibir información para prevenir el mal uso de las redes sociales.

\section{CONCLUSIONES}

Es necesario difundir más información en los jóvenes acerca de la seguridad al usar las redes sociales, ya que a pesar de que afirman conocer los riesgos la mayoría sigue publicando su ubicación y lo que hace, y no se cerciora de haber cerrado correctamente la sesión, lo que genera una situación de vulnerabilidad. 


\section{Bibliografía}

AMIPCI (23 de 01 de 2014). www.amipci.org.m. Obtenido de www.amipci.org.m: https://www.amipci.org.mx/estudios/habitos_de_internet/Estudio_Habitos_del_Interna uta_Mexicano_2014_V_MD.pdf

AMIPCI (1 de enero de 2016). amipci.org.mx. Obtenido de amipci.org.mx: https://amipci.org.mx/images/Estudio_Habitosdel_Usuario_2016.pdf

Direccion General del Sistema Estatal de Informática (26 de enero de 2014). dgsei.edomex.gob.mx. Obtenido de dgsei.edomex.gob.mx: http://dgsei.edomex.gob.mx/tecnotips/redes_sociales_y_nuestro_estado_animo

Ellen, V., Temmy, S., y Martín, V. (2014). Enseñar a los adolescentes los riesgos: Una propuesta de intervención secundaria. revistacomunicar, 124.

Forbes (15 de diciembre de 2016). forbes.com.mx. Obtenido de forbes.com.mx: http://www.forbes.com.mx/las-15-redes-sociales-mas-populares-de2016/\#gs.WgKulyY

googlemaps. (14 de diciembre de 2016). google maps. Obtenido de google maps: https://www.google.com.mx/maps/place/Gas+Del+Valle+De+Tollocan,+S.A.+De+C.V./@19 $.0215799,-$

100.0093456,17z/data=!3m1!4b1!4m5!3m4!1s0x85cd715b43710347:0x9e4b9dee2473221e!8 $\mathrm{m} 2 ! 3 \mathrm{~d} 19.0215799 ! 4 \mathrm{~d}-100.0071569$

Maria, L. C., Cristina, S., y Jesús, M. G. (2016). Recursos Tecnológicos en Contextos Educativos. Madrid: UNED.

María, L., Virginia, L., y Emilio, G. (s.f.). websatafi.com. Obtenido de websatafi.com: http://www.websatafi.com/joomla/attachments/125_REDES\%20SOCIALES\%20PUB LICAR.pdf

ONTSI (1 de diciembre de 2011). osimga.gal. Obtenido de osimga.gal: http://www.osimga.gal/export/sites/osimga/gl/documentos/d/20111201_ontsi_redes_s ociais.pdf

Roxana, M., Atilio, M., Vanina, s., y Florencia, B. (1 de septiembre de 2010). Ministerio de la Educación. Obtenido de Ministerio de la Educación: http://www.me.gov.ar/escuelaymedios/material/redes.pdf 
Universidad Católica de Valencia (11 de marzo de 2016). online.ucv.es. Obtenido de online.ucv.es: http://online.ucv.es/marketing/ventajas-y-desventajas-de-las-redessociales-en-la-educacion/ 